\title{
Importancia del perfil profesional docente en el proceso de enseñanza aprendizaje
}

Importance of the teaching professional profile in the Teaching - Learning process

Xiomara Edith Espinoza Reyes ${ }^{1}$, Ana Isabel Fernández Lara ${ }^{2}$, Mónica del Carmen Pachar Puga $^{3}$

\begin{abstract}
.
DOI: https://doi.org/10.33262/cienciadigital.v3i1.288

This bibliographical review article aims to analyze competency-based education that contains the potential to become an effective innovation plan, for which the education system is required to face change, but this approach requires a long reform process, which necessarily implies the transformation of educational actors: teachers and students, in which we are told that the mission of educating in the current reality should be organized around four lessons learned from professional and social competences, as length of a person: knowledge, know-how, knowing how to be and knowing how to be.

In view of the importance that these competences have in the development of the teaching profession and in the way in which it influences, the present investigative work will try to address the characteristics or define the professional profile of the teachers based on the evaluation of Competitiveness establishing its importance in the teaching - learning process for which I have considered translating this prototype in the Carmen Mora de Encalada School, of the Pasaje district, El Oro Province as

\footnotetext{
${ }^{1}$ Universidad Indoamérica, Facultad de Ciencias Humanas, de la Educación y Desarrollo social, Ambato, Ecuador, xespinoza_82@hotmail.com

${ }^{2}$ Universidad Indoamérica, Facultad de Ciencias Humanas, de la Educación y Desarrollo social, Ambato, Ecuador, anafernandez@uti.edu.ec

${ }^{3}$ Universidad Indoamérica, Facultad de Ciencias Humanas, de la Educación y Desarrollo social, Ambato, Ecuador, mpacharpuga@ hotmail.com
} 
part of its vision of supporting a new paradigm for training of the students and the teaching staff: a goal that, with commitment and maturity, can be achieved.

Keywords: Teacher, competency-based approach, teaching profile, teaching competences, teaching learning

\section{Resumen.}

El presente artículo de revisión bibliográfica tiene el fin de analizar la educación basada en competencia que contiene el potencial para convertirse en un plan eficaz de innovación, para lo cual se requiere que el sistema educativo se enfrente al cambio, pero este enfoque necesita de un largo proceso de reformas, lo que implica junto a la, la trasformación de los actores educativos: docentes y estudiantes, Estableciendo que en la realidad actual la misión de educar debe organizarse alrededor de cuatro ejes: el saber, el saber hacer, el saber estar y el saber ser.

En vista de la importancia que estas competencias tienen en el desarrollo de la profesión docente y en el modo en el que influye, el presente trabajo influye se tratara de abordar las características, o definir el perfil profesional de los docentes a partir de la valoración de las competitividades, estableciendo su importancia en el proceso de enseñanza - aprendizaje por lo cual he considerado plasmar este prototipo en el Colegio Carmen Mora de Encalada, del cantón Pasaje, Provincia de El Oro como parte de su visión de apoyar a un nuevo paradigma para la formación de los estudiantes y planta docente: meta que, con compromiso y madurez; se podrá lograr.

Palabras claves: Docente, enfoque basado en competencias, perfil docente, competencias docentes, enseñanza aprendizaje

\section{Introducción.}

El Perfil profesional docente ha sido un tema de estudio de muchos años y cada vez toma mayor importancia a través del tiempo por ser la parte fundamental en el desempeño del educador y en el proceso de enseñanza- aprendizaje. Se refiriere a una variable primordial que interviene en la interrelación estudiantes - docentes. Incide en la dinámica del procedimiento educativo y, por lo tanto, en su calidad (Alfaro, Gamboa, Jiménez, Pérez, Ramírez y Vargas, 2008). Se maneja el concepto de perfil docente como el de un sujeto que facilita el aprendizaje, siendo este un ente o agente de cambio social, que ha logrado competencias para enfrentar con éxito un proceso de trasferencia y adquisición de conocimientos por parte de un grupo de personas que son los estudiantes de cualquier nivel educativo o de diferentes modalidades. (Cóbar 2011).

Álvarez (2011) expresa que la educación basada en competencias contiene el potencial para convertirse en un plan eficaz tendiente a mejorar la asimilación de los conocimientos por parte del alumnado y debe ser un reto que hay que aceptar e integrar en la cultura académica. 
En este sentido, se tendría un vigoroso instrumento, con el fin de diseñar currículos innovadores, fortalecer el aprendizaje y, con ello, acortar la distancia que se ha ido abriendo entre la instrucción en los planteles educativos y la práctica profesional.

Galvis (2007) expone que se hace necesario: la creación de un perfil del enseñante que cumpla con las competencias requeridas por el profesor de hoy; que englobe las exigencias, necesidades y expectaciones que demandan los retos del sistema educativo que condicionan el papel de un educador con un perfil competente para la enseñanza - aprendizaje y a las habilidades, destrezas, rasgos de personalidad, la conformación física, el nivel de educación inherente al desempeño docente relacionados con un currículo y descripción profesional en un mundo competitivo que dote al maestro de una identidad propia

Finalmente, Tardif (2006) concibe a la competencia como un saber actuar complejo, destacando el carácter integrador, la combinación y movilización de recursos variados (internos y externos) para resolver diversas situaciones del entorno. Delors (citado en Sánchez, 2012) declara que la misión de educar en la realidad actual debe organizarse alrededor de cuatro aprendizajes de las competencias profesionales y sociales, a lo largo de la vida de una persona: el saber, el saber hacer, el saber estar y el saber ser.

Por lo que en el presente artículo investigativo se realizará un análisis de las características del perfil docente necesario para abordar el enfoque basado en competencia en el proceso de enseñanza - aprendizaje. El papel del profesor ante las demandas educativas del siglo XXI. Según expresa Rodríguez, Aguilar, Jiménez y Pérez (citado en Álvarez, 2011) la sociedad actual está en un proceso de readaptación, desde un modelo industrial a uno basado en el conocimiento. El tratado del libre comercio, la globalización y la celeridad con la que se desarrolla la tecnología de la información y comunicación, marcaron el comienzo del siglo XXI y, con ello, el cambio educativo que tiene como reto trasformar y cambiar la manera de formar individuos, de manera que se les proporcionen los elementos necesarios para vivir en una colectividad competitiva.

Por lo tanto, la educación es un proceso integral que exige actualmente un cambio urgente de un nuevo paradigma basado en un concepto de enseñanza - aprendizaje para lograr un nivel de desarrollo de las capacidades de las personas. Además, debe ser adecuado a las necesidades del entorno, a la dinámica de la ciencia, al uso de tecnología e información y a las competencias requeridas por el individuo del siglo XXI (Álvarez, 2011)

Ante esta situación el perfil docente es una de las variables fundamentales que intervienen en la interrelación estudiantes-docentes e incide en la dinámica del proceso educativo y, por tanto, en su calidad siendo rasgos específicos que caracterizan al educador para afrontar las situaciones del entorno. (Alfaro et al., 2008). Las competencias profesionales del docente. Según Sánchez (2012) el término competencia, ha tomado relieve en el ámbito educativo en 
las últimas dos décadas, el cual hace referencia a las conocimientos, habilidades, características y destrezas que debe tener el profesional que ejerce la docencia.

En ese mismo sentido, Fernández y Sánchez declaran que las competencias son el conjunto de conocimientos, habilidades, actitudes y valores que, de manera coordinada, conducen a desempeñar exitosamente una tarea. Lo que ciertamente distingue a los mejores son características no relacionadas con la concepción tradicional de inteligencia; sino con los rasgos de personalidad, las motivaciones estables o los valores personales, reflejados en pensamientos, emociones y comportamientos.

Cabe agregar que, a partir de una serie de definiciones recopiladas sobre el término, se puede extraer una síntesis de los elementos comunes en las mismas, como define Fernandez y Sánchez: Son características o atributos personales: conocimientos, habilidades, aptitudes, rasgos de carácter, conceptos de uno mismo. Están relacionados con ejecuciones que producen resultados exitosos. Se manifiestan, por tanto, en la acción. Logran resultados en diferentes contextos y, por ello, no son características estables. Son trasferibles, en el sentido de que el sujeto las puede aplicar a cualquier actividad, sector o función. Son susceptibles de ser entrenadas y desarrolladas a partir de programas de formación (2014).

Anteriormente, en el informe de la UNESCO precedida por Delors se expuso la necesidad del desarrollo de cuatro competencias en el ámbito educativo en el trascurso de la existencia de un humano aprender a conocer, aprender a hacer, aprender a vivir juntos, aprender a ser: (1) Aprender a conocer, combinando una cultura general suficientemente amplia con la posibilidad de profundizar los conocimientos en un pequeño número de materias. Lo que supone, además: aprender a aprender para poder aprovechar las posibilidades que ofrece la educación a lo largo de la vida. (2) Aprender a hacer a fin de adquirir no solo una calificación profesional sino, más generalmente, una competencia que capacite al individuo para hacer frente a gran número de situaciones y a trabajar en equipo. Pero, también, aprender a hacer en el marco de las distintas experiencias sociales o de trabajo que se ofrecen a los jóvenes y adolescentes, bien espontáneamente a causa del contexto social o nacional, bien formalmente gracias al desarrollo de la enseñanza por alternancia. (3) Aprender a vivir juntos desarrollando la comprensión del otro y la percepción de las formas de interdependencia realizar proyectos comunes y prepararse para tratar los conflictos- respetando los valores de pluralismo, comprensión mutua y paz. (4) Aprender a ser para que florezca mejor la propia personalidad y se esté en condiciones de obrar con creciente capacidad de autonomía, de juicio y de responsabilidad personal. Con tal fin, no menospreciar en la educación ninguna de las posibilidades de cada individuo: memoria, razonamiento, sentido estético, capacidades físicas, aptitud para comunicar (1996).

Al respecto Tribó plantea que mientras, el paradigma educativo tradicional, basado en la enseñanza, tendía a privilegiar el conocimiento disciplinar o saber. En cambio, el nuevo paradigma centrado en el aprendizaje concibe la educación como la integración en un todo 
de los cuatro pilares y orienta la escolarización obligatoria a la adquisición de las competencias básicas para todos los ciudadanos. Esta es la finalidad que inspira las nuevas titulaciones de la Convergencia Europea, basadas en las competencias profesionales y el aprendizaje a lo largo de la vida (2008)

También añade que la relación de reciprocidad de conocimientos varios y la capacidad de reunirlos para lograr un objetivo en común y de ponerlos en práctica de manera simultánea en la praxis profesional es la característica que, desde nuestro punto de vista, define la competencia de una persona en un ámbito profesional. Por otro lado, Perrenoud pone de manifiesto que es casi imposible conseguir los propósitos del sistema educativo, sin las competencias necesarias que requiere el educador para ejercer su labor docente. Adicionalmente, en una investigación posterior, en 2004 el mismo autor expone diez competencias consideradas prioritarias que deben tener los profesionales de la educación:

1. Organizar y animar las situaciones de aprendizaje: conocer el currículo, los aprendizajes esperados que deben alcanzar los alumnos al término de un grado o ciclo escolar, utilizar metodologías con enfoques por competencias: proyectos, casos, ABP, dilemas éticos, consignas, etc.

2. Gestionar la progresión de los aprendizajes: observar y evaluar a los alumnos en su desempeño, evaluar con un enfoque formativo. Promover la regulación de los aprendizajes.

3. Elaborar y hacer evolucionar dispositivos de diferenciación: atender la diversidad de alumnos que conforman la clase-grupo. Aplicar adecuaciones curriculares de apoyo a alumnos que lo requieran. Promover el trabajo entre pares.

4. Implicar a los alumnos en sus aprendizajes y en su trabajo: fomentar la autoevaluación, la competencia del aprendizaje permanente, negociar proyectos a trabajar, así como dar a conocer los propósitos que se persiguen con las actividades a realizar. Orientar y ayudar a los alumnos a construir un proyecto de vida.

5. Trabajar en equipo: utilizar metodologías de trabajo que desarrollen competencias, abonar al trabajo colaborativo, analizar situaciones que aquejan a la sociedad en su entorno inmediato o lejano (globalización). Promover la resolución de conflictos y la superación de situaciones que fortalezcan la unidad.

6. Participar en la gestión de la escuela: contribuir a la elaboración de un proyecto escolar que sea una herramienta organizativa funcional que oriente el trabajo del colectivo hacia el logro de los propósitos educativos.

7. Informar e implicar a los padres: consolidar el carácter social de la evaluación, al dar rendimiento de cuentas a los padres de familia de avances o dificultades en el aprendizaje de los alumnos. Promover su apoyo hacia el logro académico de sus hijos. 
8. Utilizar las nuevas tecnologías: aprovechar los recursos de la información y comunicación que pueden apoyar la educación. Crear comunidades de aprendizaje entre docentes y estudiantes, utilizando la telemática.

9. Afrontar los deberes y los dilemas éticos de la profesión: promover un ambiente de diálogo como forma de solucionar los conflictos. Prevenir la violencia en la escuela.

10. Luchar contra los prejuicios sociales, culturales, étnicos, etc. Promover valores para la sana convivencia.

11. Organizar la propia formación continua: establecer un trayecto formativo que contribuya a la mejora de la gestión pedagógica. Conformar una comunidad de aprendizaje entre los docentes de la escuela que permita disminuir las dificultades que como colectivo enfrentan (2001).

Las competencias que debe poseer el profesor del siglo XXI, coincidiendo en gran medida con la propuesta de Perrenoud, no obstante, adiciona otras como la comunicación que tiene relación con el talento para trasmitir de formas pedagógicas los contenidos en el aula, de tal manera que la información impartida la podamos trasladar y se convierta en conocimiento para los estudiantes; la relación-comunicación con los alumnos que trasciende el mero hecho de la trasmisión de conocimientos y abarca interacciones personales entre alumno-docente con el propósito de formarlos en valores y adiestrarlos en habilidades sociales con sus pares y demás miembros de la comunidad, del mismo modo, ser críticos e indagadores de los procesos de trasmisión de conocimientos

Finalmente, Tribó citado en Fernández y Sánchez (2014) expresa que un docente es "competente cuando sabe interrelacionar y coordinar de manera simultánea conocimientos de los cuatro ámbitos definidos, para aplicarlos de manera integrada a una situación profesional concreta y ha adquirido la habilidad de saber trasferir este conocimiento competencial a nuevas situaciones" (p. 4).

\section{El perfil docente basado en competencias y su influencia en el aprendizaje y rendimiento de los estudiantes.}

Para comenzar el análisis de la relación entre las competencias docentes y el rendimiento académico de los estudiantes, mencionaremos a Castro (2007) quien analiza la labor docente en la sociedad actual, y declara que mientras los educadores en su formación académica desarrollan principalmente la competencia sobre la trasmisión de conocimientos, los planes actuales constan de habilidades conceptuales, procedimentales y actitudinales, que permiten trasportar al estudiante a solucionar problemas de forma proactiva e incentivándolos a autorregular su proceso de enseñanza aprendizaje; por tal razón se debe formar a los educandos con el objeto de que desarrollen todas las capacidades como son las referentes al conocimiento, metodología, relaciones interpersonales que permitan su perfeccionamiento y actualización constante. 
Con referencia a lo anterior Tejada indica que debido la variedad de historiales particulares y profesionales que se desenrollan en diversas situaciones de instrucción emerge la disposición de determinar un perfil para ejercer la profesión educativa, que tiene relación con dos contextos: la aglomeración de una familia profesional y las competencias educativas que son constitutivas del perfil mencionado (2009).

Según se ha visto, existen cuatros competencias principales que requieren los docentes para ejercer su profesión, y cada una influye de diferente manera en el rendimiento académico, sin embargo ¿Qué es el rendimiento académico? Al respecto, autores como Lima (2012) ponen de manifiesto que el rendimiento académico no solo hace referencia a los resultados del coeficiente intelectual y actitudinal, sino también las condiciones intrínsecas del individuo, son la consecuencia del entorno sistémico del estudiante, donde son consideradas sus emociones, capacidades considerando situaciones en las que se encuentra.

Morazán define al respecto: en nuestra sociedad en los planteles educativos el desempeño académico tiene que ver la obtención de calificaciones, aprobación de módulos o en sentido contrario reprobar y obtener bajas calificaciones y en consecuencia y con esto podrá disminuir, igualar o mejorar las condiciones de vida

En referencia a los nuevos retos que pide la colectividad, la educación debe formar seres humanos con condiciones objetivas, con la destreza de conseguir, procesar y razonar la información contemporánea para después utilizar esos conocimientos. Desde esta perspectiva el rendimiento académico es un indicador del nivel de aprendizaje alcanzado por el estudiante por lo que el sistema educativo le brinda una considerable importancia, desde este escenario se convierte en una tabla imaginaria de medida para el aprendizaje logrado en el aula, que constituye el objetivo central de la educación (2013).

Como puede observarse la sociedad en los últimos 20 años ha sufrido cambios vertiginosos y cada vez más dudosos y por lo tanto debemos formar estudiantes líderes de su conocimiento, y para alcanzar este reto, quienes primero deben cambiar son los docentes, quienes con su práctica profesional y basada en competencias aumentasen las actitudes, habilidades y destrezas de cada alumno.

A continuación, vamos a examinar cómo influye cada competencia en el proceso de enseñanza y por consiguiente en el desempeño académico d cada estudiante.

El saber hacer en la práctica educativa del profesional de la educación implican el uso de técnicas, estrategias y metodologías, que él o la docente hace suyas, las lleva al aula. Además, hace comprensión sobre un cuerpo de valores, creencias y formas de hacer las cosas, para con este facilite el aprendizaje en los docentes. Es aquí donde esta competencia incide positivamente en el aumento del desempeño académico del estudiante, al docente saber trasmitir su conocimiento de forma pedagogía, didáctica, planificada y comunicativa (Morazán, 2013). 
En cuanto a la competencia de la planificación García, Loredo, y Rueda (2008) expresan que: la presencia de esta competencia pone de manifiesto cuando él o la docente domina los saberes de su materia. Además, delimita el enfoque de enseñanza (metas, filosofía, postura epistemológica y didáctica), ubica los saberes en contextos disciplinares, curriculares y sociales amplios, estructura los saberes para facilitar experiencias de aprendizaje significativo. También selecciona o desarrolla materiales didácticos, organiza y distribuye correctamente el tiempo de la instrucción, establece claramente y en acuerdo con los y las estudiantes, las reglas de convivencia, sustentadas en valores universales de respeto a los derechos humanos. Al establecer los criterios de desempeño y acreditación de la asignatura, diseña situaciones para facilitar experiencias de aprendizaje: significativo; colaborativo y autónomo, incorpora el uso de tecnologías de la información y la comunicación para apoyar los procesos de enseñanza, aprendizaje y evaluación, establece estrategias alternativas para apoyar a los alumnos, de acuerdo con sus necesidades de formación.

En este mismo orden y dirección, cabe destacar la importancia de la puesta en práctica por parte del educador del "saber estar" en el proceso enseñanza-aprendizaje debido que hay que recordar que él o la docente desempeña un papel fundamental en la vida de la o el estudiante, pues por un lado es el facilitador del aprendizaje, pero también se espera que provea a los estudiantes seguridad emocional, que atienda la diversidad y lo conduzca o guíe en lo concerniente a los comportamientos (Mordaza, 2013).

Al respecto Morazán refiere: otra competencia importante en el proceso de trasmisión y adquisición de saberes es la evaluación, debido a que es importante para apreciar la evolución del desarrollo de los estudiantes y poder modificar los planes para atender la necesidad de cada alumno

Finalmente, el docente que no ha desarrollado de forma óptima las competencias docentes impactara de forma negativa en el rendimiento académico de los alumnos y formara estudiantes con conocimientos erróneos, y con bajo valor personal y social (2013)

\section{Estudios realizados sobre el Perfil Docente Basado en Competencias en Castellón - España.}

Fernández y Sánchez sabiendo de la importancia de las competencias en el desarrollo profesional docente y su influencia en el proceso de enseñanza-aprendizaje, analizaron a 136 profesores de nueve especialidades de secundaria de la Provincia Castellón - España sobre: cuál es el grado de significancia que le otorgan los docentes a la formación por competencias, la existencia de perfiles de docentes a partir de la valoración de las competencias, y la caracterización de dichos perfiles en función de las variables personales y contextuales consideradas.

Los autores Sánchez y Fernández plantean al respecto: con base en los resultados del primer objetivo se evidenció, la importancia que dan los educadores a las competencias del saber, 
denotando que todavía la formación de los mismos está orientada hacia el modelo tradicionalista, por consiguiente, se debe reestructurar el papel del docente, con el propósito de incorporar a sus capacidades las del saber hacer, saber estar y saber. De acuerdo con los resultados obtenidos con el segundo y tercer objetivo, dan como consecuencia, que se sigue formando profesores para clases imposibles de impartir en planteles educativos inexistentes, de ahí que se deben hacer correctivos en materia formativa con el fin de lograr aumentar la importancia percibida de las competencias en este colectivo (2014).

\section{Investigación y análisis realizados sobre la Formación Profesional y competencias docentes en el estado de Tlaxcala - México.}

Carro, Hernández, Lima; Corona (2016) realizaron un estudio para medir la práctica docente y la relación con algunas variables trasversales, sin embargo, para el objeto del presente artículo se analizará los resultados y conclusiones referentes a la variable competencia basándose en las referencias establecidas por la Secretaría de Educación Pública, mediante el Acuerdo número 447 detalladas a continuación:

1. Organiza su formación continua a lo largo de su trayectoria profesional.

2. Domina y estructura los saberes para facilitar experiencias de aprendizaje significativo.

3. Planifica los procesos de enseñanza y de aprendizaje atendiendo al enfoque por competencias, y los ubica en contextos disciplinares, curriculares y sociales amplios.

4. Lleva a la práctica procesos de enseñanza y de aprendizaje de manera efectiva, creativa e innovadora a su contexto institucional.

5. Evalúa los procesos de enseñanza y de aprendizaje con un enfoque formativo.

6. Construye ambientes para el aprendizaje autónomo y colaborativo.

7. Contribuye a la generación de un ambiente que facilite el desarrollo sano e integral de los estudiantes.

8. Participa en los proyectos de mejora continua de su escuela y apoya la gestión institucional (2008).

El universo de la muestra para el estudio fue a 346 docentes de los tres niveles educativos utilizando una metodología descriptiva trasversal de corte cuantitativo. Para la recolección de los datos el instrumento utilizado fue la encuesta con la siguiente distribución: 47,9 por ciento a profesores de primaria, 30,7 por ciento a profesores de secundaria, y 21,4 por ciento a docentes de educación media superior.

Metodologia. 
El presente artículo se encuadra dentro de los siguientes tipos de investigación: cualitativa bibliográfica. La investigación se enmarca en un estudio cualitativo para analizar los conceptos, y características sobre la terminología utilizada sobre el perfil docente basado en competencias, y la interacción con el proceso de enseñanza aprendizaje, para la redacción de forma ordenada. Para la recolección de la información se utilizó el método bibliográfico con fuentes primarias y secundarias de distintos autores encontrados en repositorios digitales tales como Redalyc, Scielo, Dialnet; así como de repositorios de universidades, tesis doctorales que nos sirven para validar y verificar la información expuesta.

\section{Resultados.}

Considerando la condición y circunstancias específicas de ingreso al sistema laboral:

Tabla 1. Cuadro de doble Entrada entre los sujetos y resultados.

\begin{tabular}{|c|c|}
\hline Sujetos de estudio & Resultados \\
\hline $\begin{array}{l}\text { Profesores de } \\
\text { educación básica }\end{array}$ & $\begin{array}{l}\text { El 54,2\% adquirieron las competencias en su práctica docente } \\
\text { aprendizaje, además las capacidades relacionadas con el trabajo } \\
\text { autónomo y colaborativo también lo adquirieron en su mayoría en la } \\
\text { labor docente }(64,3 \%) \text {, no obstante, la competencia sobre organización } \\
\text { en su formación continua a lo largo de su trayectoria profesional la } \\
\text { obtuvieron en la formación académica }(39,6 \%) \text { (Carro, Hernández, } \\
\text { Lima; Corona 2016). }\end{array}$ \\
\hline $\begin{array}{l}\text { Profesores de } \\
\text { secundaria }\end{array}$ & $\begin{array}{l}\text { Un porcentaje del } 51,4 \% \text { manifiestan que obtuvieron las competencias } \\
\text { para la práctica docente en el trabajo, de la misma manera que los } \\
\text { educadores de primaria reconocen que la capacidad de trabajar en } \\
\text { forma autónoma, colaborativa, construir ambientes para el aprendizaje } \\
\text { y proyectos de mejora la desarrollaron en el ejercicio profesional más } \\
\text { que en la formación académica (Carro, Hernández, Lima; Corona } \\
2016 \text { ). }\end{array}$ \\
\hline $\begin{array}{l}\text { Profesores de } \\
\text { educación media } \\
\text { superior }\end{array}$ & $\begin{array}{l}\text { El 53,1\% obtuvieron las competencias en la labor profesional, } \\
\text { asimismo la capacidad que mayor desarrollaron en la formación } \\
\text { académica es organizar su formación continua a lo largo de su } \\
\text { trayectoria profesional con el } 42,1 \text { por ciento. En sentido opuesto, la } \\
\text { capacidad que tuvo menor porcentaje es la concerniente a construir } \\
\text { ambientes de aprendizaje autónomo y colaborativo con el 29,0 por } \\
\text { ciento (Carro, Hernández, Lima; Corona 2016). }\end{array}$ \\
\hline
\end{tabular}

Elaborado por: Grupo de investigación

Las conclusiones del estudio Carro, Hernández, Lima; Corona fueron: 
Es particularmente significativo que la formación profesional de los docentes abarca diversas disciplinas, con una tendencia a incrementar los niveles de habilitación. La especialización se distribuye en las distintas áreas del conocimiento, aunque por la naturaleza de su trabajo esta se concentra principalmente en humanidades. No obstante, lo anterior, tanto la formación docente como las competencias necesarias para la práctica pedagógica, aún hoy, no han logrado cristalizar las características de la acción educativa que teóricos como Perrenoud (2010), Lukas (Lukas \& Santiago, 2012), Malpica (2012), Meirieu (1991), han propuesto. Esto quizás tenga que ver con el tipo de formación que recibe previo a su incorporación al servicio docente.

Contrario a la finalidad que tienen las instituciones formadoras en la docencia e incluso otras instituciones, los resultados de la presente investigación afirman lo que empíricamente se había notado, la formación concreta de la docencia se obtiene en la experiencia profesional más que en la formación institucional (2016)

\section{Conclusiones.}

- El presente artículo de investigación tiene como propósito analizar el perfil profesional del docente basado en las competencias y su influencia en el proceso de enseñanza - aprendizaje como la tendencia en materia de innovación que dote al maestro de una identidad propia.

- En cuanto a la actuación del docente, se requiere de procesos continuos de reflexión e instancias formales de capacitación del profesorado que en el proceso de enseñanza -aprendizaje cumpla con las competencias requeridas por la educación del día a día, lo cual conducirá a la innovación y no adaptarse a ella y a revalorizar al maestro en un perfil basado en competencias.

- Sin embargo, cualquier innovación será en vano sin el compromiso del educador, esta formación necesita ineludiblemente de la responsabilidad del mismo, no solo para el sino también para trasferir su aprendizaje y sugerir que el perfil profesional del docente, a pesar de estar inmerso en la sociedad de la información y comunicación, todavía están enfocados en el modelo tradicionalista de impartir contenidos.

- Por esta razón es necesario formar profesionalmente a los maestros para que adquieran una variedad de competencias necesarias para que la trasmisión de conocimiento genere un aprendizaje significativo debido a la variedad de técnica, metodología y recursos utilizados para impartir clases, estableciendo vías de comunicación y relación con los estudiantes con el fin de motivarlos extrínsecamente como intrínsecamente, además se debe seleccionar al sujeto o individuo con pruebas de personalidad para determinar su vocación, motivación y cualidades internas de ser humano.

- Un educador que este en constantemente construcción del conocimiento y fortaleciendo sus competencias ejercerá de mejor manera su papel de educador, 
beneficiando al estudiante no solo en el contenido científico, sino interpersonal y social y por ende tendrá un mayor rendimiento académico.

- Por el contrario, si el profesor no ha sido formado para desarrollar de forma eficiente todas sus competencias impactará de forma negativa en el proceso de enseñanza aprendizaje, debido que impartirá contenidos erróneos que los estudiantes no podrán aplicar en su contexto.

- Por tal razón los centros educativos y gobiernos deben modificar los currículos para que el profesional de la docencia adquiera toda la competencia durante su formación académica.

- Para concluir esta difícil tarea requiere la participación de los docentes con una gran aspiración de cambiar este nuevo paradigma educativo, meta que, con sacrificio, compromiso, madurez y pasión, se podrá lograr.

\section{Referencias bibliográficas.}

Alfaro, M., Gamboa, A., Jiménez, S., Pérez, J., Ramírez, A., \& Vargas, M. (2008). Construcción del perfil profesional docente de séptimo año: respuesta a una necesidad actual. Revista Electrónica Educare, XII 08(2), 31-45. Recuperado de: http://www.redalyc.org/articulo.oa?id=194114586005

Álvarez, M. (2011). Perfil del docente en el enfoque basado en competencias. Revista Electrónica Educare, 15(1), 99-107 Recuperado de https://www.google.com/url?sa=t\&rct=j\&q=\&esrc=s\&source=web\&cd=1\&ved=0a hUKEwjA18mQn8DaAhXDjVkKHQN_AzoQFggoMAA\&url=https\%3A\%2F\%2F dialnet.unirioja.es\%2Fdescarga\%2Farticulo\%2F3683582.pdf\&usg=AOvVaw3oqnU IFcquswZ2tc2Aw2vo

Carro, A., Hernández, F., Lima, J. \& Corona, M. (2016). Formación profesional y competencias docentes en el estado de Tlaxcala. Educación, 25(49), 7-28. Recuperado de http://www.scielo.org.pe/scielo.php?script=sci_arttext\&pid=S101994032016000200001

Cobar, M. (2011). Influencia del perfil docente en el proceso de enseñanza aprendizaje de la UFG, 11(33), 47-52. Recuperado de http://ri.ufg.edu.sv/jspui/bitstream/11592/8499/1/Influencia\%20del\%20perfil\%20do cente $\% 20$ en $\% 20$ el $\% 20$ proceso $\% 20 \mathrm{de} \% 20 \mathrm{ense} \% \mathrm{C} 3 \% \mathrm{~B} 1$ anza $\% 20$ aprendizaje $\% 20 \mathrm{de}$ $\% 201 a \% 20$ UFG.pdf

Delors, J. (1996). Informe Unesco. La educación encierra un tesoro, 41-62.

Ferrández, R. \& Sánchez, L. (2014). Competencias docentes en secundaria. Análisis de perfiles de profesorado. Relieve. Revista Electrónica de Investigación y Evaluación 
Educativa,

https://www.uv.es/relieve/v20n1/RELIEVEv20n1_1.pdf

Galvis, R. V. (2007). De un perfil docente tradicional a un perfil docente basado en competencias. Acción pedagógica, 16(1), 48-57. Recuperado de https://dialnet.unirioja.es/descarga/articulo/2968589.pdf

García, J. (2011). Modelo educativo basado en competencias: importancia y necesidad. Revista Electrónica "Actualidades Investigativas en Educación", 11 (3), 1-24. Recuperado http://www.redalyc.org/html/447/44722178014/

Lima, B. (2012). Características de la vulnerabilidad familiar de las familias migrantes y su asociación con el rendimiento académico obtenido por los adolescentes de primero, segundo y tercero de bachillerato durante el año lectivo 2010 - 2011, que estudian en el colegio militar "abdón calderón" de la ciudad de cuenca". (Memoria de tesis de maestría, Universidad de Cuenca, Ecuador). Recuperado de http://dspace.ucuenca.edu.ec/bitstream/123456789/2695/1/tm4662.pdf

Morazan, S. (2013). Competencias docentes y su relación con el rendimiento académico en la asignatura de matemáticas en las instituciones de educación media del municipio de Danlí (Memoria de tesis de doctorado, Unidad Nacional Pedagógica Francisco Morazán, Guatemala) http://repositorio.upnfm.edu.hn:8081/xmlui/handle/12345678/352

OCDE (2002). Conocimientos y aptitudes para la vida. Primeros resultados del Programa Internacional de Evaluación de Estudiantes (PISA) 2000 de la OCDE. México:Santillana.

Tejada, J. (2009). Competencias docentes. Revista de currículum y formación de profesorado. Vol. 13, Núm 2. España. Pp. 1-15. Recuperado de: http://www.ugr.es/ recfpro/rev153COL1.pdf

Perrenoud, P. (2001). La formación de los docentes en el siglo XXI. Revista de Tecnología educativa, $\quad$ 14(3), 503-523. Recuperado de https://www.unige.ch/fapse/SSE/teachers/perrenoud/php_main/php_2001/2001_36. html\#copyright

Perrenoud, P. (2004). Diez nuevas competencias para enseñar: invitación al viaje (Vol. 196). Graó. Recuperado de https://www.uv.mx/dgdaie/files/2013/09/Philippe-PerrenoudDiez-nuevas-competencias-para-ensenar.pdf

Sanchez, L. (2012). Importancia de las competencias docentes según el profesorado de secundaria de la provincia de Castellón. Recuperado de 
http://repositori.uji.es/xmlui/bitstream/handle/10234/76562/-serveis-scp-publ-jfixvii-educacio-15.pdf? sequence $=1 \&$ is Allowed $=y$

Sarramona, J. (2007). Las competencias profesionales del profesorado de secundaria. Estudios sobre educación. (12), 31-40. Recuperado de:

https://dadun.unav.edu/bitstream/10171/8992/1/12\%20Estudios\%20Eb.pdf

Sarramona, J. (2011). ¿ Qué significa ser profesional docente en la actualidad?. Revista Portuguesa de Pedagogia, 427-440. Recuperado de https://digitalisdsp.uc.pt/bitstream/10316.2/5321/2/39\%20\%20Que\%20Significa\%20ser\%20Profes ional\%20Docente $\% 20 \mathrm{en} \% 20 \mathrm{la} \% 20$ Actualidad.pdf?ln=pt-pt

Tardif, J. (2006). L'évaluation des compétences. Documenter le parcours de développement. Montréal : Chenelière Éducation

Tribó, G. (2008). El nuevo perfil profesional de los profesores de secundaria. Educación XX1, (11), 183-209. Recuperado de http://www.redalyc.org/pdf/706/70601109.pdf

Zabalza, M.A. (2007). La enseñanza universitaria. El escenario y sus protagonistas. Madrid: Narcea.

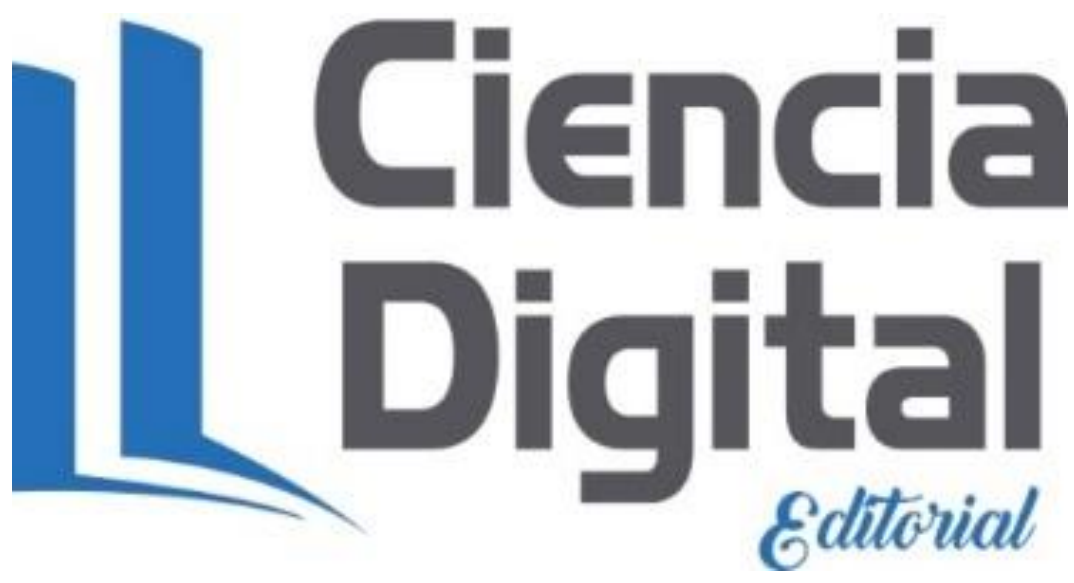




\section{Para citar el artículo indexado.}

Espinoza X, Fernández A, Pachar M. (2019). Importancia del perfil profesional docente en el proceso de enseñanza aprendizaje. Revista electrónica Ciencia Digital 3(1), 272-286.

Recuperado desde:

http://cienciadigital.org/revistacienciadigital2/index.php/CienciaDigital/article/view/288/69 $\underline{1}$

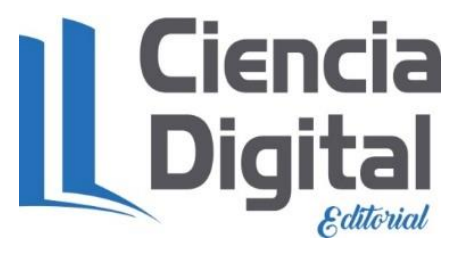

El artículo que se publica es de exclusiva responsabilidad de los autores y no necesariamente reflejan el pensamiento de la Revista Ciencia Digital.

El artículo queda en propiedad de la revista y, por tanto, su publicación parcial y/o total en otro medio tiene que ser autorizado por el director de la Revista Ciencia Digital.
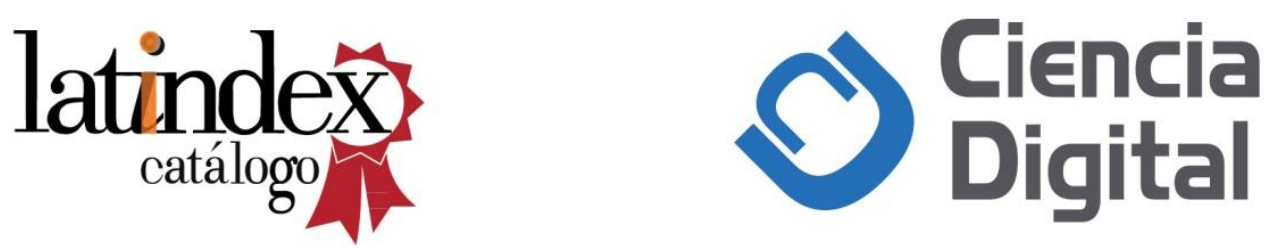